\title{
Kouass (Asilah, Maroc)
}

Campagne d'études 2015

Virginie Bridoux, Mohamed Kbiri Alaoui, Éliane Lenoir et Halima Naji

\section{(2) OpenEdition \\ Journals}

Édition électronique

URL : http://journals.openedition.org/cefr/1623

DOI : $10.4000 /$ cefr. 1623

ISSN : 2282-5703

Éditeur

École française de Rome

Référence électronique

Virginie Bridoux, Mohamed Kbiri Alaoui, Éliane Lenoir et Halima Naji, « Kouass (Asilah, Maroc) », Chronique des activités archéologiques de l'École française de Rome [En ligne], Maghreb, mis en ligne le 20 septembre 2016, consulté le 30 avril 2019. URL : http://journals.openedition.org/cefr/1623 ; DOI : $10.4000 /$ cefr. 1623

Ce document a été généré automatiquement le 30 avril 2019.

(c) École française de Rome 


\section{Kouass (Asilah, Maroc)}

Campagne d'études 2015

Virginie Bridoux, Mohamed Kbiri Alaoui, Éliane Lenoir et Halima Naji

\section{NOTE DE L'AUTEUR}

La mission est co-dirigée par V. Bridoux (chargée de recherche à l'UMR 8546 « AOROC ») et M. Kbiri Alaoui (enseignant-chercheur à l'INSAP, Rabat, Maroc). Les co-responsables des études en cours, que nous remercions pour leur contribution tout au long de l'année, sont : N. André (architecte-ingénieur de recherche à l'USR 3155 IRAA/CNRS-AMU, Aix-enProvence) ; B. Clavel (chargé de recherche au CNRS, UMR 7209, Muséum national d'Histoire naturelle, Paris) ; É. Grisoni (chercheur associé à l'UMR 5608, TRACES, Toulouse) ; H. Hassini (conservateur du site archéologique de Lixus) ; A. Ichkhakh (inspecteur des monuments historiques et des sites, Essaouira, Maroc) ; T. Jullien (membre associé à l'UMR 7298, Aix-en-Provence / Marseille Université, CNRS, LA3M, Aixen-Provence) ; É. Lenoir (ancienne chargée de recherche à l'UMR 8546, CNRS-ENS Paris Ulm) ; H. Naji (conservateur à la division d'inventaire et de documentation du patrimoine, Direction du patrimoine culturel, Rabat). L'équipe de Kouass remercie ceux qui ont porté intérêt à ses travaux et lui ont rendu visite en 2015 dans les réserves de l'INSAP où sont conservés le mobilier et la documentation scientifique : A. Akerraz (directeur de l'INSAP), A. Alaoui (directeur du Patrimoine culturel), A. Arharbi (conservateur des sites de Banasa et Thamusida), A. Mikdad (directeur adjoint de l'INSAP) et E. Papi (professeur à l'Université de Sienne, Italie).

1 La troisième campagne du deuxième programme quadriennal «Recherches archéologiques franco-marocaines à Kouass ", effectuée en 2015, a été consacrée à la poursuite de l'analyse du mobilier et de la documentation scientifique issus des fouilles menées depuis 2009 sur ce site de la façade nord-atlantique du Maroc.

2 Pour rappel, les grandes phases d'occupation ont pu être ainsi déterminées :

- la période islamique $\left(\mathrm{XII}^{\mathrm{e}}-\mathrm{XV}^{\mathrm{e}} \mathrm{s}\right.$.),

- la période maurétanienne récente ( ${ }^{\mathrm{er}} \mathrm{s} . \mathrm{av} . \mathrm{J} . \mathrm{C}$. .), 
- la période maurétanienne moyenne (III ${ }^{\mathrm{e}}-\mathrm{II}^{\mathrm{e}} \mathrm{s}$. av. J.-C.),

- la période maurétanienne ancienne ( $\mathrm{V}^{\mathrm{e}}-\mathrm{IV}^{\mathrm{e}} \mathrm{s}$. av. J.-C.),

- la période maurétanienne archaïque (antérieure au $\mathrm{V}^{\mathrm{e}} \mathrm{s}$. av. J.-C.).

Il s'agissait d'étudier principalement deux catégories de céramique d'époque maurétanienne, à savoir la céramique dite de «type Kouass » et la céramique commune. La classification de la céramique islamique a par ailleurs été précisée, en particulier celle des récipients culinaires et de ceux servant à la préparation des aliments, travail qui fera prochainement l'objet d'une publication. Il en résulte des corpus descriptifs élaborés pour chaque catégorie de matériel, présentés par formes/variantes, illustrés par un catalogue de planches $^{1}$ et incluant les conclusions préliminaires relatives aux contextes de découverte, dont nous fournissons ici un premier aperçu.

\section{La céramique de « type Kouass » (fig. 1)}

La céramique de «type Kouass » a été initialement identifiée par M. Ponsich et considérée par ce dernier comme une imitation de céramique campanienne produite localement à la suite de la découverte sur le site d'un four contenant des « débris de vases et de ratés »².

Fig. 1 - Principales formes de céramique de «type Kouass ».

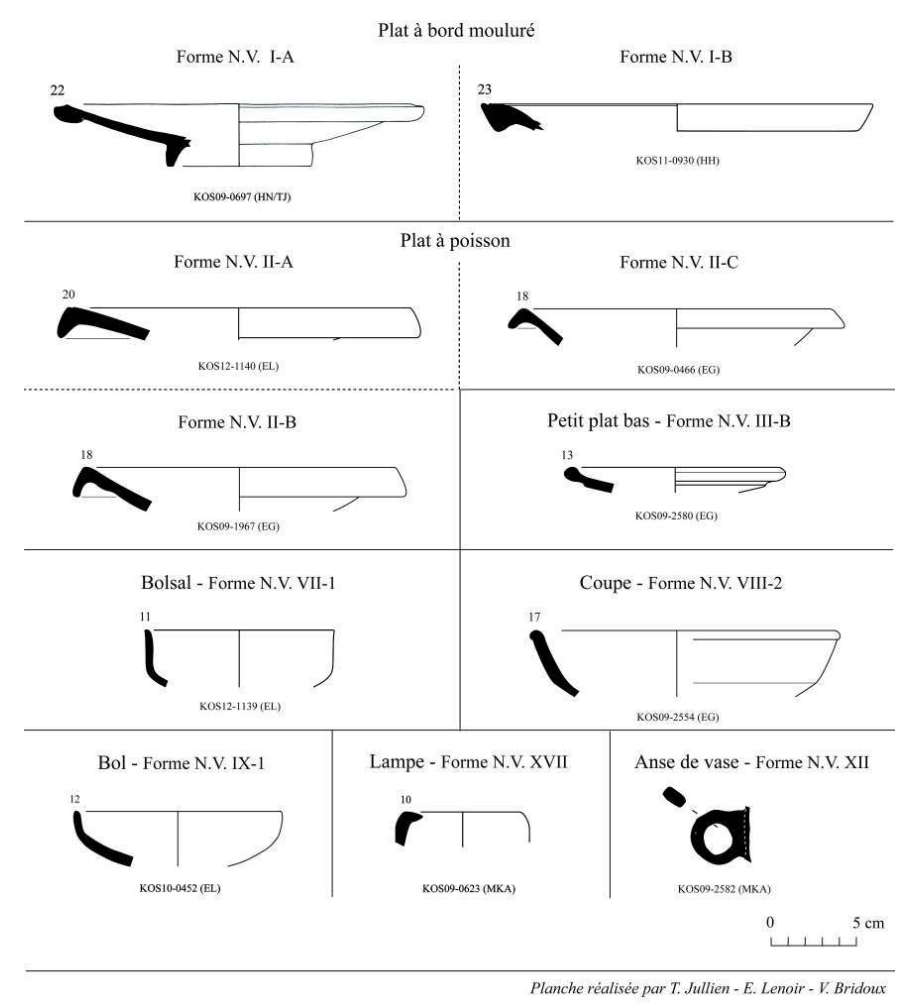

T. Jullien, E. Lenoir, V. Bridoux.

Elle est en fait diffusée de part et d'autre du Détroit de Gibraltar et est notamment bien documentée sur les sites de la baie de Cadix. La publication des ensembles de cette région par A. M. Niveau de Villedary y Mariñas en 2003 précise les caractéristiques générales de cette production à engobe brun/rouge qui s'inspire en réalité en premier lieu du répertoire des céramiques à vernis noir hellénistiques. Les céramiques de "type Kouass » constituent désormais un fossile directeur pour la datation des niveaux archéologiques 
puisqu'elles s'avèrent fabriquées afin de pallier la fin des importations attiques jusqu'à l'arrivée massive des céramiques campaniennes (fin $\mathrm{IV}^{\mathrm{e}}-\mathrm{II}^{\mathrm{e}} \mathrm{s}$. av. J.-C.) ${ }^{3}$. Elles reproduisent plus précisément les formes fonctionnelles de cette vaisselle, notamment le service de table. Céramiques majoritairement lisses, elles sont parfois décorées d'estampilles (des palmettes ou des rosettes).

La campagne menée en 2015 contribue à enrichir le répertoire des formes et variantes attestées sur le site à la suite du réexamen du mobilier issu des fouilles de M. Ponsich ${ }^{4}$. L'examen du lot des céramiques de "type Kouass » provenant des fouilles menées depuis 2009 a porté sur 148 fragments correspondant à un total approximatif de 119 individus dont un est archéologiquement complet. Malgré le caractère fragmentaire de ce lot, 11 principales formes d'objets ont pu être identifiées sur la base de la typologie dressée par A. M. Niveau de Villedary y Mariñas et ici indiquée par l'abréviation N.V. ${ }^{5}$

6 Les plats sont de loin les plus fréquents et parmi ce groupe la forme la plus récurrente est le plat à poisson, dont 10 variantes sont documentées (N.V. II). On dénombre également deux types de plats à bord mouluré (N.V. I) dont l'un est archéologiquement complet; trois variantes de petits plats bas (N.V. III) que A. M. Niveau de Villedary y Mariñas considère, à partir de contextes ibériques bien déterminés, comme des objets à usage rituel ou des objets dédiés à la libation ${ }^{6}$; enfin de petits fragments de bords appartenant manifestement d'une part à des plats à bord concave et lèvre bombée (N.V. V), d'autre part à un plat de forme large et basse à bord légèrement évasé et à carène (N.V. VI).

7 Les bols de forme N.V. IX sont également bien représentés avec la présence de 10 variantes. S'ajoutent cinq variantes de bolsals (N.V. VII), trois variantes de coupes (N.V. VIII), les restes d'un vase profond à anse en anneau de forme N.V. XII dont l'usage est rattaché aux rites de libation ${ }^{7}$ et une lampe de forme N.V. XVII- variante C. Cette lampe globulaire de type grec est pour la première fois documentée à Kouass, mais on notera que le réexamen du matériel des fouilles de M. Ponsich fait état d'une autre forme de lampe de forme ouverte (forme N.V. XVI) ${ }^{8}$.

\section{La céramique commune d'époque maurétanienne (fig. 2 et 3 )}

8 L'étude de la céramique commune d'époque maurétanienne a pour objectif l'élaboration d'une typo-chronologie fondée sur l'analyse du répertoire des formes attestées et de leur association dans les niveaux archéologiques avec du mobilier dont la datation est relativement bien précisée. 
Fig. 2 - Principales formes de céramique commune d'époque maurétanienne : formes 1 à 6.

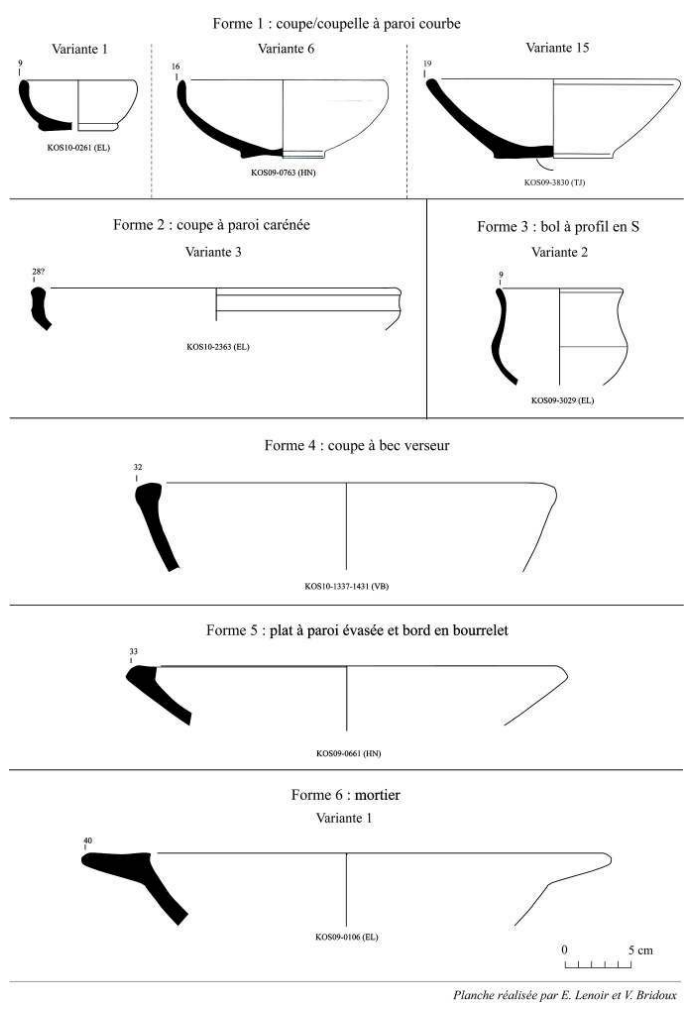

E. Lenoir, V. Bridoux

Fig. 3 - Principales formes de céramique commune d'époque maurétanienne : formes 7 à 8 .
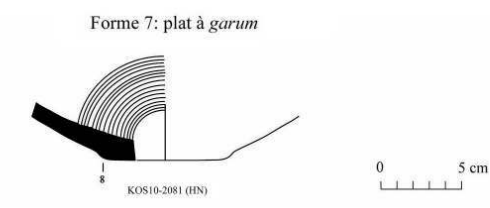

Forme 8 : lampe

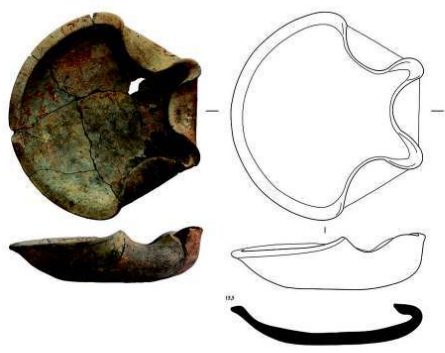

$\operatorname{Kos}(93.3838$ (T)

$\stackrel{2 \mathrm{~m}}{\mathrm{c}}$

Planche réalisée par T. Jullien, E. Lenoir, V. Bridoux

T. Jullien, E. Lenoir, V. Bridoux. 
En 2015, le travail a porté notamment sur les objets de formes ouvertes dont le nombre minimum d'individus s'élève à 538. Huit principaux types de formes ont été définis, généralement à partir de l'observation des bords dans la mesure où trop peu d'objets complets ont pu être restitués et où peu de fonds ont pu être associés aux bords ${ }^{9}$. Les objets de forme identifiable, représentés par 405 individus, se répartissent comme suit selon les contextes stratigraphiques :

- 158 individus dans des couches superficielles ou dans les remblais des fouilles des années 1960

- 45 individus dans des contextes dont la datation demeure incertaine

- 53 individus dans des niveaux de réoccupation d'époque islamique

- 18 individus dans des niveaux datables de la période maurétanienne sans que l'on puisse préciser davantage la chronologie

- 94 individus dans des niveaux datables de la période maurétanienne ancienne

- 27 individus dans des niveaux datables de la période maurétanienne moyenne

- 10 individus dans des niveaux datables de la période maurétanienne récente.

Sauf mention contraire, les formes recensées sont présentes dans les strates d'époque maurétanienne ancienne à récente. Il s'agit :

- de coupes/coupelles à paroi courbe plus ou moins épaisses et profondes (forme 1), très majoritaires (320 individus, répartis en 15 variantes). On notera que le groupe de la variante 15 est fortement prédominant (114 individus)

- de coupes peu profondes à paroi carénée (forme 2), peu représentées (18 individus, répartis en 3 variantes). Cette forme est absente des niveaux d'occupation d'époque maurétanienne ancienne

- de bols à profil en « $\mathrm{S}$ » et panse globulaire (forme 3), très peu nombreux (8 individus, répartis en 2 variantes

- de grandes coupes (forme 4) à bec verseur et lèvre en " $T$ », représentées par 21 individus. Cette forme, déjà identifiée dans le répertoire de la céramique peinte ${ }^{10}$ (forme 15) et parmi le mobilier des fouilles des années $1960^{11}$, n'avait pas été documentée jusque là comme étant munie d'un bec verseur. Cette forme est vraisemblablement dérivée de la tradition phénicienne et l'on note d'ailleurs que tous les individus en céramique commune exhumés en contextes stratigraphiques préromains, soit plus de la moitié des objets, sont issus de niveaux d'époque maurétanienne ancienne. Les objets de ce type en céramique peinte provenant de contextes stratigraphiques sont également datables de la période maurétanienne ancienne

- de six plats à paroi évasée et bord en bourrelet (forme 5), dont seuls deux ont été exhumés en stratigraphie et s'avèrent appartenir à des niveaux datables de l'époque maurétanienne ancienne

- de mortiers (forme 6) à bord horizontal, triangulaire ou tombant, dont on dénombre 16 individus, répartis en 5 variantes

- de plats à fond plat considérés par M. Ponsich comme des plats à garum (forme 7), extrêmement rares ( 2 individus) et mal documentés en contexte stratigraphique

- de lampes (forme 8) de tradition punique en forme de coupelle peu profonde à becs pincés (variante 1), bien attestées dans les niveaux d'occupation d'époque maurétanienne ancienne mais aussi dans les niveaux du $\mathrm{I}^{\mathrm{er}} \mathrm{s}$. av. J.-C. (époque maurétanienne récente). Il est intéressant de constater l'association dans ces niveaux maurétaniens récents de fragments de lampes de tradition punique et de type tardo-républicain (variante 2). 
corpus réalisés cette année à partir de ces études viennent alimenter la connaissance du répertoire et de l'évolution des céramiques de production locale ou régionale d'époque maurétanienne. Ils seront intégrés au catalogue général du mobilier en cours d'élaboration sur lequel pourra se fonder l'analyse comparative du matériel recueilli sur les sites voisins de Maurétanie occidentale, mais aussi de l'Ibérie, en vue de préciser notamment la place de Kouass au sein de l'aire économique et culturelle de l'Extrême Occident méditerranéen.

\section{BIBLIOGRAPHIE}

Bridoux et al. 2015 = V. Bridoux, M. Kbiri Alaoui, N. André, É. Grisoni, A. Ichkhakh, T. Jullien, É. Lenoir et H. Naji, Kouass (Asilah, Maroc). Campagne d'étude 2014, Chronique des activités archéologiques de l'École française de Rome [En ligne], mis en ligne le 03/07/2015, https://cefr.revues.org/79.

Kbiri Alaoui $2007=$ M. Kbiri Alaoui, Revisando Kuass (Asilah, Marruecos) : Talleres cerámicos en un enclave fenicio, púnico y mauritano, Valence, 2007 (Saguntum Extra 7).

Niveau de Villedary y Mariñas 2003 = A. M. Niveau de Villedary y Mariñas, Las cerámicas gaditanas " tipo Kuass », bases para el analisis de la bahía de Cádiz en época púnica, Madrid, 2003.

Niveau de Villedary y Mariñas 2008 = A. M. Niveau de Villedary y Mariñas, La cerámica « Tipo Kuass ", dans D. Bernal Casasola, A. Ribera i Lacomba (dir.), Cerámicas hispanorromanas : un estado de la cuestión, Cádiz, 2008, p. 245-262.

Ponsich 1969 = M. Ponsich, Les céramiques d'imitation : la campanienne de Kouass, dans AEA, XLII, 1969 , p. 56-80.

\section{NOTES}

1. La réalisation de l'ensemble des planches a été confiée à $T$. Jullien et É. Lenoir à partir des dessins et des mises au net effectués par les différents membres de l'équipe.

2. Ponsich 1969, p. 57.

3. Niveau de Villedary y Mariñas 2003, p. 279-281.

4. Pour les caractéristiques des céramiques issues des fouilles de M. Ponsich à Kouass, voir Kbiri Alaoui 2007, p. 171-188.

5. L'analyse des contextes de découverte de la céramique de «type Kouass » et du matériel qui lui est associé est actuellement en cours.

6. Niveau de Villedary y Mariñas 2008, p. 248.

7. Niveau de Villedary y Mariñas 2008, p. 252.

8. Kbiri Alaoui 2007, p. 148, fig. 155.

9. Les remarques concernant la description des pâtes sont en cours de traitement. L'étude devra notamment permettre de déterminer les équivalences au sein de la céramique commune mais aussi les correspondances avec les autres catégories, en premier lieu avec les objets appartenant au répertoire de la céramique peinte.

10. Cf. Bridoux et al. 2015. 
11. Elle est également attestée à Lixus. Kbiri Alaoui 2007, fig. p. 133, objet sans numéro de catégorie, en bas à droite.

INDEX

Mots-clés : Maghreb, établissement maurétanien, établissement médiéval, céramique, faune institutions Institut national des sciences de l'archéologie et du patrimoine (INSAP, Rabat, Maroc), Ministère des Affaires étrangères et du Développement international (Paris), École française de Rome

\section{AUTEURS}

\section{VIRGINIE BRIDOUX}

UMR 8546 «AOROC » (CNRS / ENS Paris Ulm) - virginie.bridoux[at]ens.fr

\section{MOHAMED KBIRI ALAOUI}

INSAP (Rabat, Maroc) - medkbirialaoui[at]hotmail.com

\section{ÉLIANE LENOIR}

Ancienne chargée de recherche, UMR 8546 « AOROC » (CNRS / ENS Paris Ulm)

\section{HALIMA NAJI}

Division d'inventaire et de documentation du patrimoine, Direction du Patrimoine (Rabat, Maroc) - halinaji[at]yahoo.fr 